\title{
Combining Coq and Gappa for Certifying Floating-Point Programs ${ }^{\star}$
}

\author{
Sylvie Boldo ${ }^{1,2}$, Jean-Christophe Filliâtre ${ }^{2,1}$, and Guillaume Melquiond ${ }^{1,2}$ \\ 1 INRIA Saclay - Île-de-France, ProVal, Orsay, F-91893 \\ 2 LRI, Université Paris-Sud, CNRS, Orsay, F-91405 \\ Sylvie.Boldo@inria.fr \\ Jean-Christophe.Filliatre@lri.fr \\ Guillaume.Melquiond@inria.fr
}

\begin{abstract}
Formal verification of numerical programs is notoriously difficult. On the one hand, there exist automatic tools specialized in floatingpoint arithmetic, such as Gappa, but they target very restrictive logics. On the other hand, there are interactive theorem provers based on the LCF approach, such as Coq, that handle a general-purpose logic but that lack proof automation for floating-point properties. To alleviate these issues, we have implemented a mechanism for calling Gappa from a Coq interactive proof. This paper presents this combination and shows on several examples how this approach offers a significant speedup in the process of verifying floating-point programs.
\end{abstract}

\section{Introduction}

Numerical programs typically use floating-point arithmetic [1]. Due to their limited precision and range, floating-point numbers are only an approximation of real numbers. Each operation may introduce an inaccuracy and their total contribution is called the rounding error. Moreover, some real operations may not be available as sequences of floating-point operations, e.g., infinite sums or integrals. This introduces another inaccuracy called the method error. Both errors make it somehow complicated to know what floating-point programs actually compute with respect to the initial algorithms on real numbers.

One way to proceed is to give a program a precise specification of its accuracy. Generally speaking, a specification explains what can be expected from the result given facts about the inputs. Typically, it bounds the sum of both rounding and method errors. For example, the specification for a function float_cos defined on the double type of floating-point numbers may be the following: ${ }^{3}$

$$
\forall x: \text { double, }, \quad|x| \leq 2 \pi \Rightarrow\left|\frac{\cos (x)-\text { float_cos }_{-}(x)}{\cos (x)}\right| \leq 2^{-53}
$$

\footnotetext{
* This research was partially supported by projects ANR-05-BLAN-0281-04 "CerPAN" and ANR-08-BLAN-0246-01 "F $\oint$ ST".

${ }^{3}$ Note that $\pi / 2$ cannot be represented by a floating-point number, therefore $\cos (x)$ cannot be zero.
} 
Such an inequality is typically proved using pen and paper. It can be deduced from the specification of each floating-point operation and the mathematical properties of the cosine function. Such proofs are notoriously difficult and errorprone.

Ideally, the code of a software would be analyzed by a certification tool, which would answer whether it is correct or not. In order to increase the confidence in the answer (and therefore in the software), the tool may rely on formal methods. Obviously, such a tool is not conceivable, but we aim at making this process as automatized as possible.

In this paper, we will describe how we have combined existing tools to help in the process of formally certifying numerical codes. From a user point of view, the first step is to annotate the source code with the specifications of the software. This annotated code is sent to a first tool that produces proof obligations corresponding to the correctness of the software. The examples of this paper are $\mathrm{C}$ programs and we are using the Caduceus tool (Section 2.1). By computing weakest preconditions based on the code and the annotations, it generates theorem statements to be verified by automated theorem provers or proof assistants.

Some theorems, hopefully most of them, will be automatically discharged. For instance, numerical properties may be discharged by the Gappa tool (Section 2.3), which is very efficient at proving bounds, especially on rounding errors. But Gappa only tackles the floating-point fragment of a program, so properties that involve more than just floating-point arithmetic may not be handled.

The remaining theorems will have to be manually handled by the user in a proof assistant with a suitable floating-point formalization. The paper focuses on the use of Coq for this task (Section 2.2). When using a proof assistant, the user issues tactics to split the goal into simpler subgoals. Examples of such tactics are logical cut, case analysis, or induction. This will become tedious if the user has to repeat this process until all the subgoals are discharged, which may require a high number of explicit proof steps. Especially frustrating is the fact that, once simplified, the subgoals may fit into specific logic fragments, which some tools, such as Gappa, could handle automatically outside Coq.

In order to benefit from Gappa inside Coq, we have implemented a mechanism for calling the tool from an interactive proof. From a technical point of view, Gappa is called as an external prover. This does not weaken the confidence in Coq formal proofs since Gappa produces a proof trace that is checked by Coq (Section 4).

This combination of Coq and Gappa does not radically change the way to tackle rounding and method errors. It simply eases the use of traditional approaches in a formal setting. C programs illustrating this point are given in Section 3. The combination of all these tools (Caduceus, Coq, Gappa) makes it possible to formally verify a source code while benefiting from automation.

There have been previous work on formally proving numerical components (especially hardware ones) while relying on automated tools. Among them, the certification of the IEEE-compliance [1] of a gate-level design for the Pentium Pro processor used Forte, a combination of two model checkers and a lightweight theorem prover [2]. Another work made the ACL2 theorem prover interact with 
a VHDL verification tool in order to prove the correctness of a hardware multiplier [3]. While not at the source-code level, two other proof assistants have been thoroughly used for verification of floating-point properties: both PVS and HOL Light provide some automation for performing error analysis $[4,5]$.

\section{Caduceus, Coq, and Gappa}

\subsection{Verification of C Programs}

The Why platform ${ }^{4}$ is a set of tools for deductive verification of Java and C programs [6]. In this paper, we only focus on verification of $\mathrm{C}$ programs but the results would apply to Java programs as well. The verification of a given $\mathrm{C}$ program proceeds as follows. First, the user specifies requirements as annotations in the source code, in a special style of comments. Then, the annotated program is fed to the tool Caduceus [7], which is part of the Why platform, and verification conditions (VCs for short) are produced. These are logical formulas whose validity implies the soundness of the code with respect to the given specification. Finally, the VCs are discharged using one or several theorem provers, which range from interactive proof assistants such as Coq to purely automatic theorem provers such as Alt-Ergo [8]. The workflow is illustrated on Figure 1.

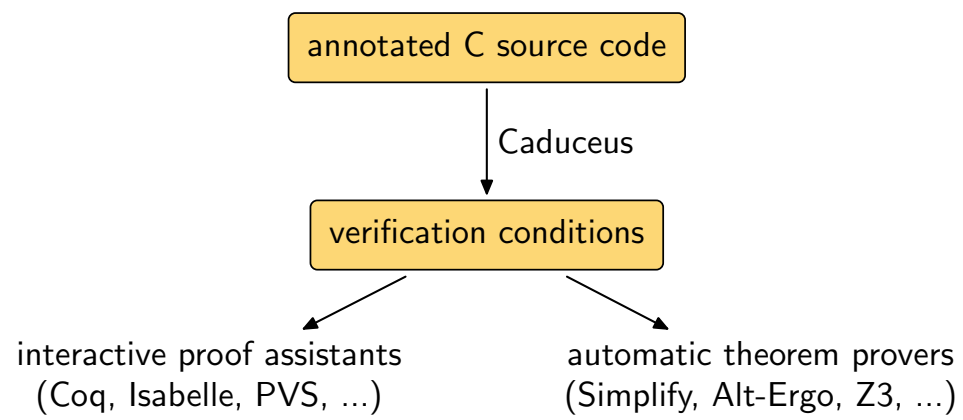

Fig. 1. The Caduceus tool.

Annotations are inserted in $\mathrm{C}$ source code using comments with a leading $@$. They are written in first-order logic and re-use the syntax of side-effect free $\mathrm{C}$ expressions. For instance, here is a code excerpt where an array $t$ is searched for a zero value.

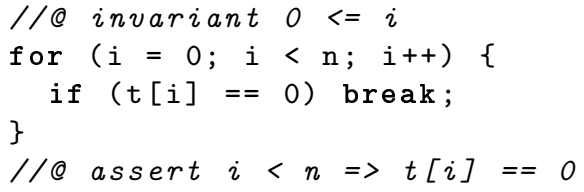

4 Available at http://why.lri.fr/. 
The for loop is given a loop invariant, as in traditional Hoare logic [9]. (In that case, the invariant could be found automatically.) A loop invariant typically generates two VCs: one to show that it holds right before the loop is entered; and one to show that it is preserved by the loop body. In this example, an assertion is also manually inserted right after the loop, which results in a VC for this program point. Additional VCs are produced to establish the safe execution of the code, i.e., that the program does not perform any division by zero or any array access out of bounds. In this example, a $\mathrm{VC}$ requires to show that $\mathrm{t}$ [i] is a legal array access, which may or may not be provable depending on hypotheses regarding $\mathrm{t}$ and $\mathrm{n}$.

Verification with Caduceus is modular: each function is given a contract and proved correct with respect to the contracts of the functions it calls. ${ }^{5}$ For instance, a partial contract for a function sorting an array of integer could be

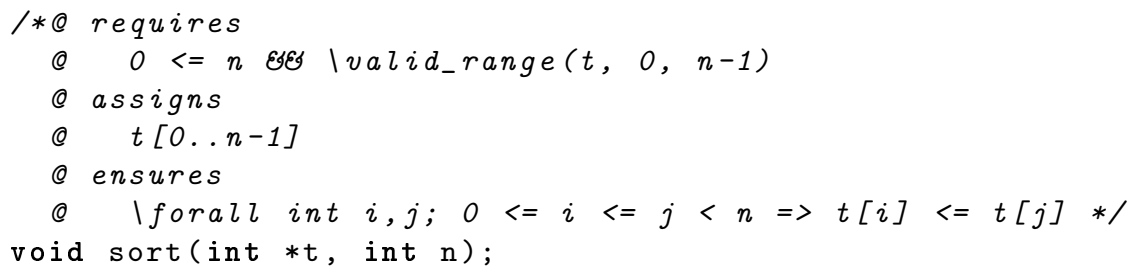

The contract contains three clauses. Keyword requires introduces a precondition, that is a property assumed by the function and proved at the caller site. In this example, it states that $\mathrm{n}$ is nonnegative and that all indices from 0 to $\mathrm{n}-1$ in $t$ can be safely accessed. Conversely, keyword ensures introduces a postcondition, that is a property provided by the function, right before it returns. Here it states that the array is sorted in increasing order. ${ }^{6}$ Finally, keyword assigns introduces the memory locations possibly modified by the function, which means that any other memory location is left unchanged by a call to this function. Here, it states that only the array elements $\mathrm{t}[i]$ for $0 \leq i<\mathrm{n}$ are possibly assigned.

Caduceus handles a large fragment of ANSI C, with the notable exception of pointer casts and unions. It handles floating-point arithmetic, using a model where each floating-point number is seen as a triple of real numbers [10]. The first component is the floating-point number itself, as it is computed. The second component is the real number that would have been computed if roundings were not performed. The third component is a ghost variable attached to the floating-point number and which represents the ideal value that the programmer intended to compute. Annotations are written using real numbers only, and the three components of a floating-point variable $\mathrm{x}$ can be referred to within annotations: $x$ itself stands for the first component; lexact $(x)$ for the second one; and $\backslash \operatorname{model}(\mathrm{x})$ for the third one. Thus the user can refer to the rounding error as the difference between the first two, and to method error as the difference between the last two. Examples are given in Section 3 .

5 That means we only establish partial correctness of recursive functions.

${ }^{6}$ For the specification to be complete, the postcondition should also state that the array is a permutation of its initial value. It can be done, but is omitted here for the sake of simplicity. 
Since the general-purpose automatic provers do not support this model of floating-point arithmetic, we have formalized it in the Coq proof assistant.

\subsection{The Coq Proof Assistant}

The Coq proof checker $[11,12]$ is a proof assistant based on higher-order logic. One may express properties such as "there exists a function which has such and such properties" or "every relation that verifies such hypothesis has a certain property" and check proofs about these. Proofs are built using tactics (such as applying a theorem, rewriting, computing, etc.). A Coq file contains the statement of lemmas and their proofs as a sequence of tactics in the Coq language.

The Coq standard library contains an axiomatization of real numbers [13]. Few automation is provided to reason about real numbers. As a consequence, the proof of a typical lemma such as $0<1-2^{-52}$ is already a few lines long:

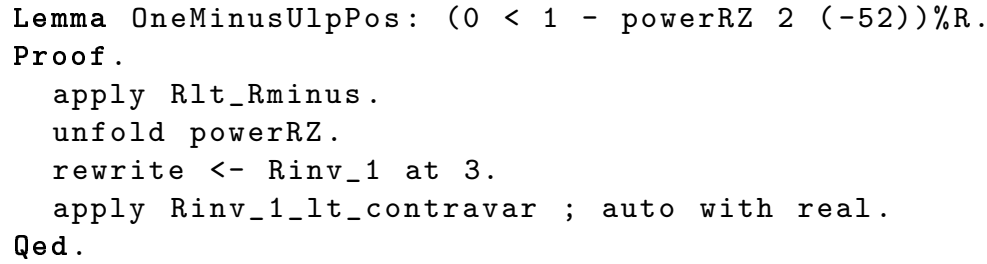

The proof is done backward, by transforming the conclusion until it trivially derives from the hypotheses. This proof starts by applying the theorem Rlt_Rminus (line 3 ) since $0<1-2^{-52}$ is a consequence of $2^{-52}<1$. The definition of powerRZ is then unfolded (line 4) so that $2^{-52}$ is converted to $\left(2^{52}\right)^{-1}$. We replace 1 by $1^{-1}$ (theorem Rinv_1, line 5 ). At this point, the goal has become $\left(2^{52}\right)^{-1}<1^{-1}$. After applying theorem Rinv_1_lt_contravar (line 6), the remaining goals are $1<2^{52}$ and $1 \leq 1$, which are solved automatically by the tactic auto (line 6 ).

A high-level formalization of floating-point arithmetic $[14,15]$ is also available in Coq. A floating-point number is a pair of integers $(m, e)$ which represents the real number $m \times 2^{e}$. The value of the mantissa $m$ and the exponent $e$ are bounded according to the floating-point format. For example, in IEEE-754 double-precision format [1], the pair verifies $|m|<2^{53}$ and $-1074 \leq e$. This library ${ }^{7}$ contains a large number of floating-point definitions and theorems and has been used to prove many old and new properties [16].

Most floating-point proofs rely on computations on real numbers, such as deciding $0<1-2^{-52}$ or bounding method error. Such goals can be addressed using the interval tactic [17]. This reflexive tactic, based on interval arithmetic, decides inequalities by bounding real expressions thanks to guaranteed floatingpoint arithmetic. Once done with method error, the user is left with VCs related to rounding errors, which Gappa is typically designed for.

\footnotetext{
7 Available at http://lipforge.ens-lyon.fr/www/pff/.
} 


\subsection{The Gappa Tool}

Gappa $^{8}$ is a tool dedicated to proving arithmetic properties on numerical programs $[18,19]$. Given a logical proposition expressing bounds on mathematical real-valued expressions, Gappa checks that it holds. The following is such a proposition and below is its transcription in Gappa's input language.

$$
\begin{gathered}
\forall x, y \in \mathbb{R}, \quad|x| \leq 2 \wedge y \in[1,9] \Rightarrow x \times x+\sqrt{y} \in[1,7] \\
\{|\mathrm{x}|<=2 \text { 八y in }[1,9] \rightarrow \mathrm{x} * \mathrm{x}+\operatorname{sqrt}(\mathrm{y}) \text { in }[1,7]\}
\end{gathered}
$$

In order to verify the proposition, Gappa first analyzes which expressions may be of interest. Then it tries to enclose them in intervals by performing a saturation over its library of theorems on interval arithmetic, forward error analysis, and algebraical identities. Gappa stops when it reaches enclosures small enough to be compatible with the right-hand side of the proposition or when the saturation does no longer improve the enclosures.

Once Gappa has verified the proposition, it generates a formal proof. To increase confidence, this proof script can then be mechanically checked by an independent proof system, such as Coq or HOL Light. ${ }^{9}$

If Gappa fails to prove the proposition, the user can suggest to the tool that it should perform a bisection - splitting input intervals until the proposition holds on each sub-intervals - or augment the library of theorems with new mathematical identities. Gappa will then assume these equalities hold; they will appear as hypotheses of the generated formal proof.

Expressing Floating-Point Programs. In addition to universally-quantified variables on $\mathbb{R}$, basic arithmetic operators $(+,-, \times, \div \sqrt{\cdot})$, and numerical constants, Gappa expressions can also contain rounding operators. The integerpart functions, $\lfloor\cdot\rfloor$ and $\lceil\cdot\rceil$, are instances of such operators. Since the IEEE-754 standard [1] mandates that "a floating-point operator shall behave as if it was first computing the infinitely-precise value and then rounding it so that it fits in the destination floating-point format", having appropriate rounding operators is sufficient to express the computations of a floating-point program.

The following script is similar to the previous one, but all the expressions are now as if they had been computed in single precision with rounding to nearest (tie-breaking to even mantissa).

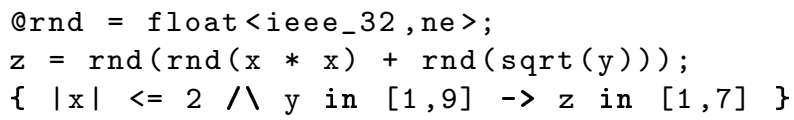

Note that Gappa only manipulates expressions on real numbers. As a consequence, infinities and NaNs (Not-a-Numbers) are no part of this formalism:

\footnotetext{
8 Available at http://lipforge.ens-lyon.fr/www/gappa/.

9 The generated proofs depend on a library of facts written for the target system. Currently, this formalization has been proved for Coq only.
} 
rounding operators return a real value and there is no upper bound on the magnitude of the input numbers. This means that NaNs and infinities will not be generated nor propagated as they would in IEEE-754 arithmetic.

We can, however, use Gappa to prove that a given code does not produce any of these exceptional values. Indeed, if one proves that a Gappa-rounded value is smaller than the biggest floating-point number in the working format, then the actual IEEE-754 computation is guaranteed not to overflow, by definition of overflow. Therefore, in order to check that computations in the previous example are overflow-safe, one can run Gappa on the following script: ${ }^{10}$

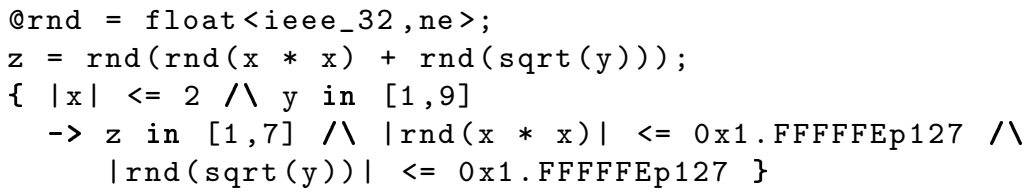

The absence of infinities and NaNs is not a deficiency, as reasoning about them is usually done by case analysis. This can be easily performed using Coq traditional tactics. For the cases without infinities and NaNs, which are the complicated ones, the Gappa tactic applies.

Verifying Accuracy. While the previous examples show that Gappa can bound ranges of floating-point variables, this is only a small part of its purpose. This tool was designed to prove bounds on computation errors, which also happen to be real-valued expressions. Let us assume that the developer actually needed the infinitely-precise result $M_{z}=x^{2}+\sqrt{y}$. Is the computed result $z$ sufficiently close to this ideal value $M_{z}$ ? This can be answered by bounding the absolute error $z-M_{z}::^{11}$

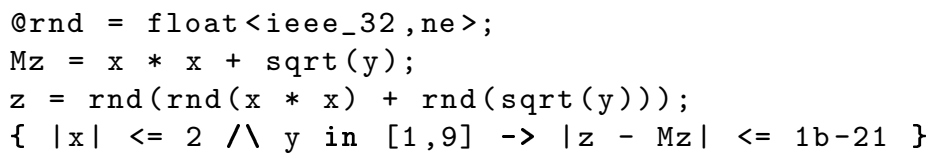

For the sake of simplicity, $M_{z}$ has the same operations as $z$, but without rounding. This is not a requirement, as Gappa is also able to bound errors when $M_{z}$ is a completely different expression. Note also that Gappa is not limited to absolute errors; it can handle relative errors in a similar way, which is especially important when proving floating-point properties.

\section{Proving Floating-Point Programs}

Before describing the inner working of the Coq-Gappa combination, we illustrate its use on the verification of three typical floating-point programs.

$\overline{10}$ The number $0 \times 1$.FFFFFE127 is the biggest finite floating-point numbers for IEEE754 single-precision format, written with the notation of the standard of the ISO C language (1999).

11 The number $1 \mathrm{~b}-21$ means $2^{-21}$, which is almost the optimal upper bound on the specified absolute error. 


\subsection{Naive Cosine Computation}

The first example is an implementation of the cosine function for single-precision floating-point arithmetic. To present a complete Coq proof, we have simplified the function by removing its argument-reduction step. Thus, input $x$ is required to have already been reduced to a value close to zero; only the polynomial evaluation has to be performed. The specification of the function states that, for $|x|$ smaller than $2^{-5}$, the computed value \result is equal to $\cos (x)$ up to $2^{-23}$.

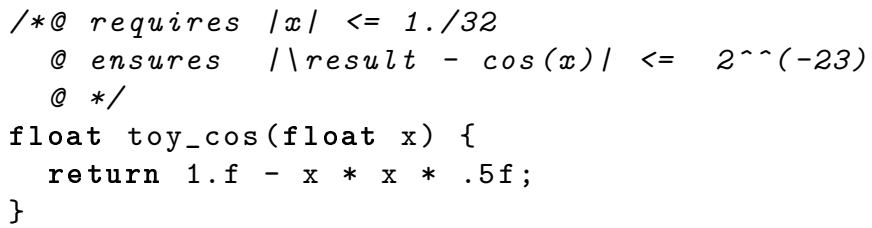

Note that $2^{-23}$ is a tight bound on the error of this function. It ensures that the computed result is one of the floating-point numbers close to the mathematical value $\cos (x)$.

Given this annotated $\mathrm{C}$ code, Caduceus generates a VC stating the accuracy of the result, which can be formally proved with the Coq script below. ${ }^{12}$

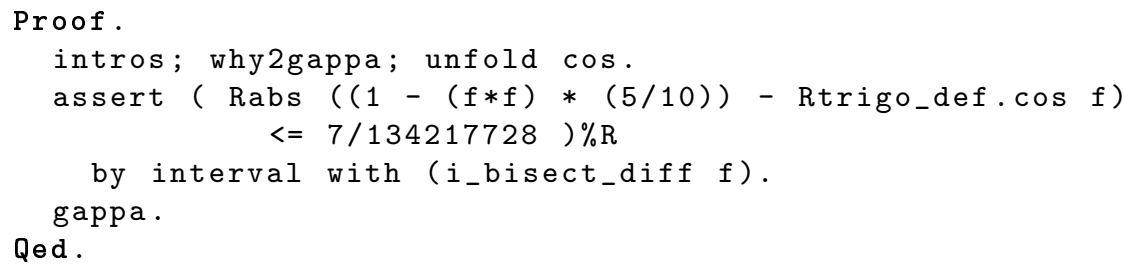

The first part of the proof script (line 2) turns the goal into a user-friendly form: the why2gappa tactic cleans the goal by expanding and rewriting some Caduceus-specific notations. At this point, assuming that $\circ(\cdot)$ is the rounding operation from a real number to the nearest single-precision floating-point number, the user has to prove the following goal:

$$
\begin{aligned}
& \forall x: \text { float }, \quad|x| \leq \frac{1}{32} \Rightarrow \\
& \quad|\circ(\circ(1)-\circ(\circ(x \times x) \times \circ(5 / 10)))-\cos (x)| \leq 2^{-23} .
\end{aligned}
$$

As would be done with a pen-and-paper verification, the formal proof of this goal starts by computing and proving a bound on the method error. Since the polynomial is chosen so that the computed result is close to the cosine, the method error is known beforehand. A typical way to obtain a polynomial approximation and its error is to use a computer algebra system.

12 The Coq script is reproduced verbatim. In particular, some terms are obfuscated due to Coq renaming them to prevent conflicts. So $f$ designates in fact the variable $x$; and Rtrigo_def.cos is the name of the cosine function in Coq's standard library. 
Here, the method error is smaller than $7 \times 2^{-27}$. So we are asserting this property in Coq (lines 3 and 4) and we prove it (line 5 ). The assertion is proved by the interval tactic [17]. Its option i_bisect_diff tells the tactic to recursively perform a bisection on the interval enclosure $\left[-2^{-5}, 2^{-5}\right]$ of $x$, until a first-order interval evaluation of the method error $(1-(x \times x) \times(5 / 10))-\cos (x)$ gives a compatible bound on all the sub-intervals.

Once the assertion is proved and hence available as an hypothesis, the user has to prove the following property:

$$
\begin{aligned}
\forall x: & \text { float }, \quad|x| \leq \frac{1}{32} \Rightarrow \\
& |(1-(x \times x) \times(5 / 10))-\cos (x)| \leq 7 \cdot 2^{-27} \Rightarrow \\
& |\circ(\circ(1)-\circ(\circ(x \times x) \times \circ(5 / 10)))-\cos (x)| \leq 2^{-23} .
\end{aligned}
$$

This is achieved by the gappa tactic (line 6). It calls Gappa and then uses the Coq script that the tool generates in order to finish the proof. Note that the Gappa tool takes advantage of the inequality proved by the interval tactic as it knows nothing about the cosine.

\subsection{Discretization of a Partial Differential Equation}

The second example is a numerical code about acoustic waves by F. Clément [20]. Given a rope attached at its two ends, a force initiates a wave, which then undulates according to the following mathematical equation:

$$
\frac{\partial^{2} u(x, t)}{\partial t^{2}}-c^{2} \frac{\partial^{2} u(x, t)}{\partial x^{2}}=0 .
$$

The value $u(x, t)$ gives the position of the rope at the abscissa $x$ and the time $t$. It is discretized both in space and time with steps $(\Delta x, \Delta t)$. The result is a matrix $p$ of size $n i \times n k$ where $p[i][k]=p_{i}^{k}$ is the position of the rope at the abscissa $i \times \Delta x$ and the time $k \times \Delta t$. The matrix $p$ is computed by the following piece of code [21], where a is an approximation of an exact constant $A$ derived from $c, \mathrm{ni}$, and nk:

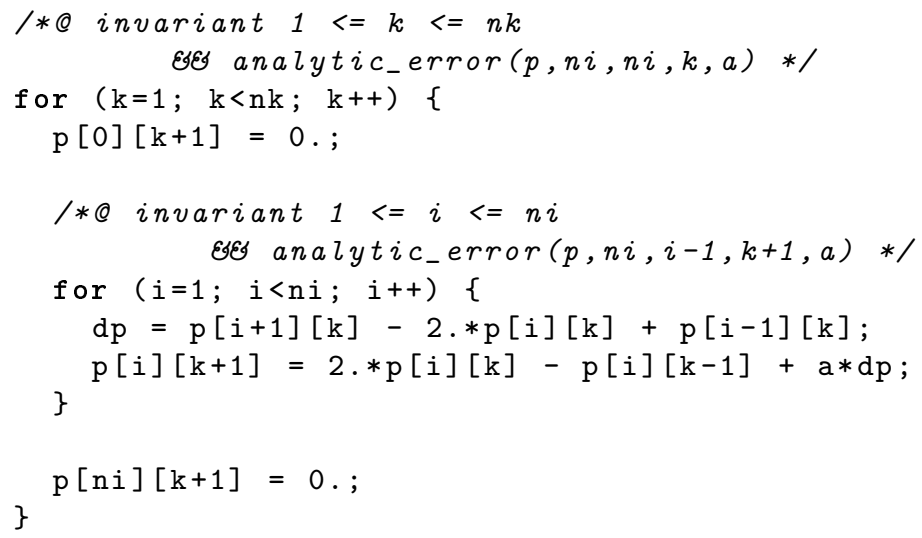


The predicate analytic_error states the exact analytical expression of the rounding error. It also states that the rounding error of a single iteration is smaller than a known value. More precisely, it bounds the absolute value of

$$
\varepsilon_{i}^{k+1}:=p_{i}^{k+1}-\left(2 p_{i}^{k}-p_{i}^{k-1}+A \times\left(p_{i+1}^{k}-2 p_{i}^{k}+p_{i-1}^{k}\right)\right) .
$$

Under some hypotheses on $A$ and the ranges of $\left(p_{i}^{k}\right)$, we could prove that $\left|\varepsilon_{i}^{k+1}\right| \leq 85 \times 2^{-52}$ (and a similar property concerning the initialization of the $p_{i}^{1}$ ) [21]. The original Coq proof amounts to 735 lines of tactics. Thanks to the gappa tactic, we were able to

- improve the result: we now have the formal proof that $\left|\varepsilon_{i}^{k+1}\right| \leq 80 \times 2^{-52}$;

- drastically cut off the size of the proof script: the 735 lines of tactics reduce to 10 .

This is a tremendous improvement. Not only is the new proof script dramatically shorter and simpler to write, but it is also more amenable to future changes and maintenance. Indeed, if the program is to be modified in such a way that the error slightly increases, the initial proof would be completely broken and only a small part could be re-used. Using Gappa, the situation is different: while the statement of the theorem would change, the proof would probably be robust enough to remain valid.

\subsection{Preventing Overflows}

Another type of proof that greatly benefits from automation is overflow proofs. Typically, one wants to guarantee that no overflows happen. To do so, it is usually sufficient to bound the program inputs. The resulting VCs are especially tedious to prove. As a consequence, the bounds are often over-estimated in order to simplify the demonstrations. This is the case for the following example. This program computes an accurate discriminant using Kahan's algorithm [22]. The accuracy is measured in ulps (unit in the last place), which is the distance between two consecutive floating-point numbers. The discriminant algorithm relies on the exactmult function which computes the rounding error of a multiplication.

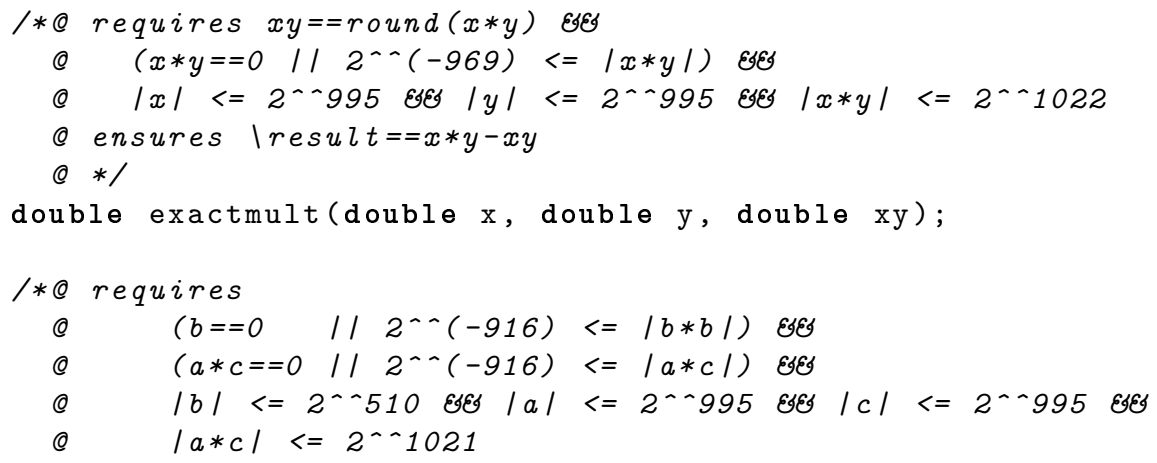




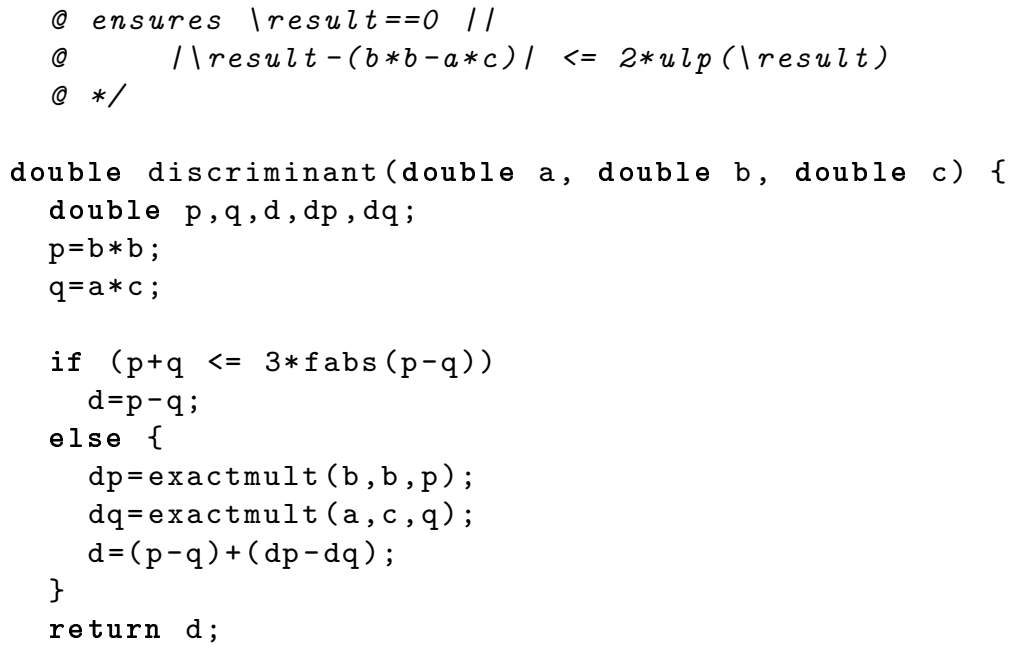

The formal proofs for this program (including overflows) have been presented in $[23,24]$. Here we only focus on the overflows of the discriminant; we do not care about the exactmult function.

All the overflow proofs were first done prior to the gappa tactic. For seven proof obligations, it took more than 420 lines of Coq. Using the tactic, the proofs reduce to 35 lines (about 5 lines per theorem). The Coq compilation time, however, is about 5 times greater. ${ }^{13}$ Nevertheless, the profit is clear in the verification process as the time for developing the proof overwhelms the time for compiling it.

It is also interesting to note that the specification is also improved. The hypothesis $|a \times c| \leq 2^{1020}$ in the original proof [24] was too strong; we proved instead that $|a \times c| \leq 2^{1021}$ is sufficient to guarantee that no overflows occur. The proof was not modified at all after changing the annotations. This means that the automation is sufficient to use exactly the same proof when modifying slightly the specification. This is really worthwhile for proof maintenance.

\section{Implementation Details}

The gappa tactic is part of the standard V8.2 Coq distribution. ${ }^{14}$ It relies on Gappa, which is an external stand-alone tool and comes with its own library of Coq theorems.

Figure 2 describes the process of performing a formal certification of a $\mathrm{C}$ program using Coq and Gappa. Starting with an annotated C program, Caduceus generates VCs corresponding to the specification of this $\mathrm{C}$ code. Lots of these proof obligations can be discharged by automatic provers. The most complicated

13 Should Coq only check proofs generated by Gappa instead of embedding them, the compilation time would be equivalent. See end of Section 4.

14 Available at http://coq.inria.fr/. 


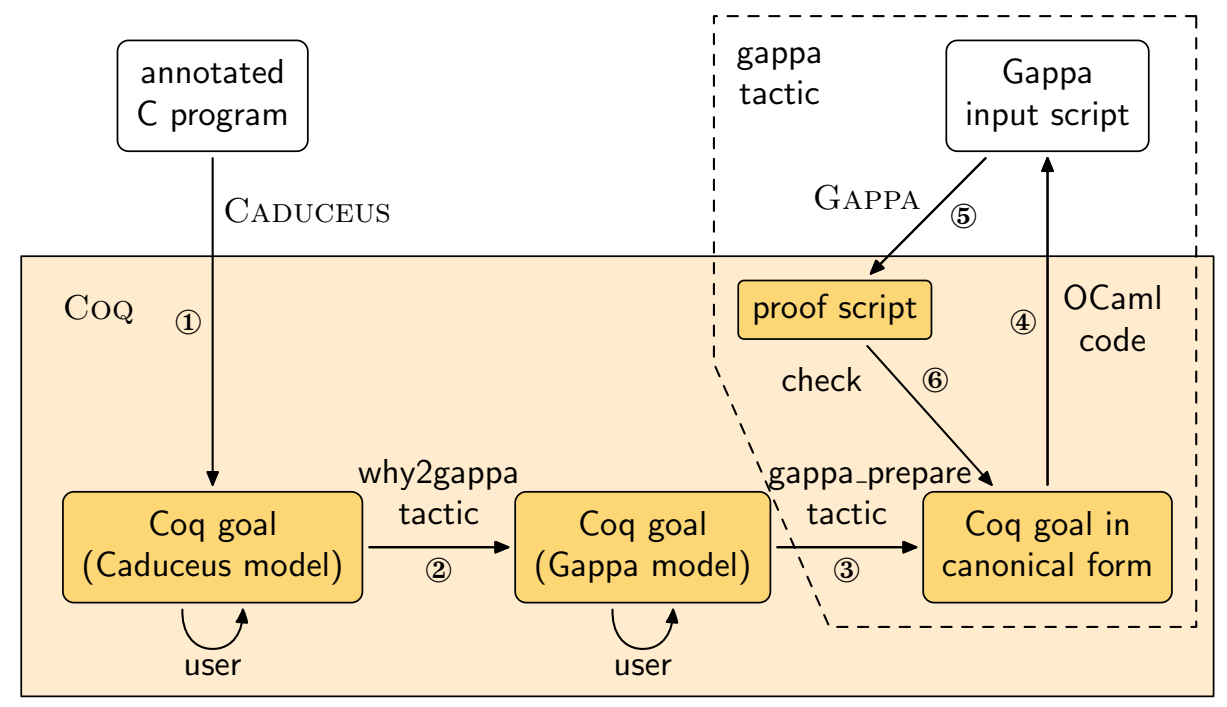

Fig. 2. Dataflow in the Coq and Gappa combination

ones, especially those involving floating-point properties, are left to the user. The Caduceus tool therefore generates template Coq scripts (Step (1)), and the user has to fill in the blanks.

At this point, the Coq goals are expressed in the floating-point model of Caduceus. As usual with a proof assistant, the user issues tactics in order to split the goal into simpler subgoals that can be handled automatically. If one subgoal is in the scope of the Gappa tool, the user can proceed as follows.

First of all, the goal has to be translated to the floating-point model of Gappa. Stored in an auxiliary library, some theorems state that both models are equivalent. In particular, if a floating-point number is the closest to a real number in the Caduceus model, then it is the result of a rounding function in the Gappa model, and reciprocally. The why2gappa tactic automatically applies these theorems to rewrite the goal and its context (Step (2)). It also unfolds the Caduceus model of floating-point numbers, with its floating-point, exact, and model parts. At this point, the goal is made of inequalities between real-valued expressions potentially containing rounding operators matching Gappa's ones.

Now, the user can launch the Gappa tool to finish the proof, thanks to a single call to the gappa tactic (Steps (3), (4), (5), and (6)). During Step (4), some OCaml code embedded into Coq reads the goal and outputs a text file suitable for Gappa. This code then runs Gappa and asks for a Coq script of the result (Step (5)). Another OCaml code loads this script into Coq, checks it, and generates the corresponding $\lambda$-term, and uses it to finish the proof (Step (6)).

This process will succeed only if the type of the Gappa $\lambda$-term matches exactly the Coq goal of the user. Otherwise, Coq would rightfully complain that the overall proof is not well-typed. For instance, the user goal could mention 
the inverse $x^{-1}$ of a variable, while the generated proof would consider $1 / x$ instead. Although equal, these two terms are not convertible, so type-checking would fail. Rather than transforming the generated script afterward, we decided to transform the goal beforehand. The gappa prepare tactic (Step (3), called internally by the gappa tactic, makes sure that the goal will not leave any margin of interpretation to the OCaml code nor to Gappa.

This subtactic is written in Ltac, the tactic language embedded into Coq and available to user scripts. It transforms all the hypotheses and the goal so that they are enclosures of the form $m_{1} \cdot 2^{e_{1}} \leq \operatorname{expr} \leq m_{2} \cdot 2^{e_{2}}$, with $m_{1}$, $e_{1}, m_{2}$, and $e_{2}$ explicit integers. Moreover, the expr part should only contain the basic arithmetic operators $(+,-, \times, \div, \sqrt{\cdot}$, and $|\cdot|)$, rounding operators, identifiers, and constants $\left(m \cdot 2^{e}\right.$ or $\left.m \cdot 10^{e}\right)$. For instance, if a proposition is $|\exp (x)+5 \times y| \leq 3 / 8$, the tactic will generalize $\exp (x)$ to a fresh identifier $e$ everywhere. Then it will replace the proposition by the (equivalent yet not convertible) proposition $0 \cdot 2^{0} \leq\left|e+\left(5 \cdot 2^{0}\right) \times y\right| \leq 3 \cdot 2^{-3}$.

In order to transform the propositions, the tactic could perform some patternmatching to find all the sub-terms that look unadapted and apply rewriting theorems to them. This method is easy to implement but slow, as a huge number of rewriting operation may be needed, especially for constants. (For instance, the real number 11 is implicitly stored by Coq as $1+(1+1) \times(1+(1+1) \times(1+1))$.) Instead, the tactic builds an inductive object that represents the syntax tree of the expressions. Some Coq functions (defined in the logic language, not in the tactic language) then implement the previous transformations. We have proved they generate a syntax tree whose evaluation as a real-valued expression gives the same result as the previous expression. Hence applying this single theorem is enough to get a suitable goal. In other words, the tactic simplifies the goal by convertibility and reflexivity $[25,17]$, which is both time- and space-efficient.

Step (4) is then trivial: the OCaml code just has to select the propositions that are enclosures, to visit the nodes of their simplified syntax trees, and to produce the corresponding Gappa script. If Gappa succeeds in verifying the script (Step (5)), the OCaml code can then load the produced proof and have Coq check it. It takes only a few seconds for the gappa tactic to reach this point after it is called.

We, however, wanted Coq not only to check the generated proof, but also to embed it into the current user $\lambda$-term. Therefore, the gappa tactic, while calling an external prover, does produce a complete Coq proof of the goal. Unfortunately, Coq is unable to deal with two scripts at once. So the tactic first launches a separate Coq session that produces a $\lambda$-term in the context of Gappa's libraries. Then it runs another separate session to get a $\lambda$-term with fully-qualified names and no notations. This last $\lambda$-term can finally be loaded in the original user session, without interfering with user-defined names and notations. This incurs a noticeable slowdown for the user. It could be fixed in two ways: enhance Coq so that other scripts can be checked in the same session, or enhance Gappa so that it directly produces a plain $\lambda$-term. Embedding the proof script into the user proof hardly increases the confidence though, since the script has already 
been checked by Coq. So, Step (6) could be reduced to type-checking the Gappa proof, creating an axiom with its type, and applying this axiom to the goal. This would ensure that the gappa tactic takes a few seconds only, without having to modify either Coq or Gappa.

Lastly, note that the gappa tactic accesses only a small part of Gappa's features. Indeed, when using the tool directly, the user can pass hints regarding properties of the problem, such as mathematical identities, to guide it. As long as the goals do not need any particular user hint, the tactic is as powerful as the tool.

\section{Conclusion}

We have presented an integration of the Gappa automated prover in the Coq proof assistant. This greatly eases the verification process of numerical programs. As shown with realistic examples, the gappa tactic significantly reduces the size of Coq proofs, and improves their maintainability. This tactic is part of the V8.2 Coq standard distribution.

This paper focuses on $\mathrm{C}$ programs verified with the Caduceus tool. However, this approach is generic enough to apply to other verification tools, such as Frama- $\mathrm{C}^{15}$ for $\mathrm{C}$ programs or Krakatoa for Java programs [6]. Indeed, our work builds upon the Why platform, which provides a common backend for Caduceus, Frama-C/Jessie, and Krakatoa. Said otherwise, any verification technology using Why to produce Coq verification conditions can benefit from the gappa tactic.

The current gappa tactic does not encompass all the features of the Gappa tool. As explained before, there is no way to pass hints to Gappa, such as interval bisection or equalities. Moreover, while the tool can infer enclosures for variables and expressions, the tactic does not offer a way to query them. This feature would relieve the user from the burden of guessing logical cuts.

The Gappa tool is limited to a small logical fragment dedicated to floatingpoint arithmetic, and so is the gappa tactic. A more ambitious perspective is to integrate Gappa to a state-of-the-art SMT solver such as Alt-Ergo [8]. This would result in more VCs discharged automatically but also in more automation when invoked from Coq.

\section{References}

1. Microprocessor Standards Subcommittee: IEEE Standard for Floating-Point Arithmetic. IEEE Std. 754-2008 (August 2008) 1-58

2. O'Leary, J., Zhao, X., Gerth, R., Seger, C.J.H.: Formally verifying IEEE compliance of floating-point hardware. Intel Technology Journal (Q1) (February 1999) $1-10$

3. Reeber, E., Sawada, J.: Combining ACL2 and an automated verification tool to verify a multiplier. In Manolios, P., Wilding, M., eds.: 6th International Workshop on the ACL2 Theorem Prover and its Applications. (August 2006) 63-70

\footnotetext{
${ }_{15}$ Available at http://www.frama-c.com/.
} 
4. Carreño, V.A., Miner, P.S.: Specification of the IEEE-854 floating-point standard in HOL and PVS. In: HOL95: 8th International Workshop on Higher-Order Logic Theorem Proving and Its Applications, Aspen Grove, UT (September 1995)

5. Harrison, J.: A machine-checked theory of floating-point arithmetic. In Bertot, Y., Dowek, G., Hirschowitz, A., Paulin, C., Théry, L., eds.: TPHOLs'99: 12th International Conference on Theorem Proving in Higher Order Logics. Volume 1690 of Lecture Notes in Computer Science., Springer-Verlag (September 1999) $113-130$

6. Filliâtre, J.C., Marché, C.: The Why/Krakatoa/Caduceus Platform for Deductive Program Verification. In: 19th International Conference on Computer Aided Verification. Volume 4590 of Lecture Notes in Computer Science., Springer (July 2007) $173-177$

7. Filliâtre, J.C., Marché, C.: Multi-prover verification of C programs. In Davies, J., Schulte, W., Barnett, M., eds.: 6th International Conference on Formal Engineering Methods. Volume 3308 of Lecture Notes in Computer Science., Seattle, WA, USA, Springer (November 2004) 15-29

8. Conchon, S., Contejean, E., Kanig, J., Lescuyer, S.: CC(X): Semantical Combination of Congruence Closure with Solvable Theories. In: 5th International Workshop on Satisfiability Modulo Theories (SMT 2007). Volume 198-2 of Electronic Notes in Computer Science., Elsevier Science Publishers (2008) 51-69

9. Hoare, C.A.R.: An axiomatic basis for computer programming. Communications of the ACM 12(10) (1969) 576-580,583

10. Boldo, S., Filliâtre, J.C.: Formal Verification of Floating-Point Programs. In: 18th IEEE International Symposium on Computer Arithmetic, Montpellier, France (June 2007) 187-194

11. Bertot, Y., Castéran, P.: Interactive Theorem Proving and Program Development. Coq'Art: The Calculus of Inductive Constructions. Texts in Theoretical Computer Science. Springer Verlag (2004)

12. The Coq Development Team: The Coq Proof Assistant Reference Manual - Version V8.2. (2008) http://coq.inria.fr/.

13. Mayero, M.: The Three Gap Theorem (Steinhauss conjecture). In: TYPES'99. Volume 1956., Springer-Verlag LNCS (2000) 162-173

14. Daumas, M., Rideau, L., Théry, L.: A generic library of floating-point numbers and its application to exact computing. In: 14th International Conference on Theorem Proving in Higher Order Logics, Edinburgh, Scotland (2001) 169-184

15. Boldo, S.: Preuves formelles en arithmétiques à virgule flottante. $\mathrm{PhD}$ thesis, École Normale Supérieure de Lyon (November 2004)

16. Boldo, S.: Pitfalls of a Full Floating-Point Proof: Example on the Formal Proof of the Veltkamp/Dekker Algorithms. In: 3rd International Joint Conference on Automated Reasoning (IJCAR), Seattle, USA (August 2006) 52-66

17. Melquiond, G.: Proving bounds on real-valued functions with computations. In Armando, A., Baumgartner, P., Dowek, G., eds.: 4th International Joint Conference on Automated Reasoning. Volume 5195 of Lecture Notes in Artificial Intelligence., Sydney, Australia (August 2008) 2-17

18. de Dinechin, F., Lauter, C., Melquiond, G.: Assisted verification of elementary functions using Gappa. In: ACM Symposium on Applied Computing, Dijon, France (2006) 1318-1322

19. Melquiond, G.: De l'arithmétique d'intervalles à la certification de programmes. PhD thesis, École Normale Supérieure de Lyon, Lyon, France (November 2006)

20. Bécache, E.: Étude de schémas numériques pour la résolution de l'équation des ondes. ENSTA (September 2003) 
21. Boldo, S.: Floats \& Ropes: a case study for formal numerical program verification. In: 36th International Colloquium on Automata, Languages and Programming. Lecture Notes in Computer Science, Springer-Verlag (July 2009)

22. Kahan, W.: On the Cost of Floating-Point Computation Without ExtraPrecise Arithmetic (November 2004) http://www.cs.berkeley.edu/ $\sim_{\text {wkahan/ }}$ Qdrtcs.pdf.

23. Boldo, S., Daumas, M., Kahan, W., Melquiond, G.: Proof and certification for an accurate discriminant. In: 12th IMACS-GAMM International Symposium on Scientific Computing, Computer Arithmetic and Validated Numerics, Duisburg, Germany (September 2006)

24. Boldo, S.: Kahan's algorithm for a correct discriminant computation at last formally proven. IEEE Transactions on Computers 58(2) (February 2009) 220-225

25. Boutin, S.: Using reflection to build efficient and certified decision procedures. In: Theoretical Aspects of Computer Software. (1997) 515-529 